\title{
Understanding chronic heart failure
}

\author{
Matthew Fenton, Michael Burch
}

Arch Dis Child 2007;92:812-816. doi: 10.1136/adc.2005.086397

The key principles of chronic heart failure and the development of clinical management strategies are described. The physiological changes in chronic heart failure and the clinical management of children with heart failure are considered, but the treatment of heart failure related to congenital heart disease or the intensive care management of heart failure are not mentioned as both topics require consideration in their own right. A greater understanding of the maladaptive responses to chronic heart failure has enabled targeted therapy to be introduced with consequent improvement in symptoms, reduction in hospitalisation and lower mortality.

See end of article for authors' affiliations

Correspondence to Michael Burch, Department of Cardiology, Great Ormond Street Hospital, London, UK:

burchm@gosh.nhs.uk

Accepted 5 January 2007 th n 1628 William Harvey published his theory on the nature of the cardiovascular system in a book entitled An anatomical study of the motion of the heart and of the blood in animals. Harvey hypothesised how the heart propelled the blood in a circular course through the body. This work underpins the current concepts involved in cardiovascular understanding to this day and the concept of heart failure, defined as the inability of the heart to eject sufficient blood to sustain the metabolic needs of the body, is based on Harvey's pioneering study.

Over the last 50 years our understanding of heart failure has continued to develop and the paradigms we use for understanding cardiac pathophysiology have changed. These now include the mechanical nature of chambers, blood vessels and valves and also extend to the understanding of neurohormonal control, cellular and molecular mechanisms, genetics and immunology. Each of these areas of research has their individual focus, but they are also inextricably linked, providing valuable information and potential targets for therapy. For example, if we take "pump failure" to be the paradigm underpinning our understanding, it seems reasonable that $\beta$-blockade would not be considered to be an appropriate therapy due to its negatively inotropic nature. However, we now know that the sympathetic overdrive produced during chronic heart failure is detrimental and $\beta$ blockers are beneficial.

Initially, this review will consider the key principles of chronic heart failure and the development of clinical management strategies will be described. In particular, the scientific basis for outpatient management of children with heart failure will be discussed. This latter section derives from experience within the specialist heart failure clinic at Great Ormond Street Hospital, London. In this clinic, the majority of children with heart failure have dilated cardiomyopathy. However, this article will focus on the effect (heart failure) rather than the cause (myocardial failure). We will not discuss treatment of heart failure related to congenital heart disease or intensive care management of heart failure as both topics require consideration in their own right.

\section{UNDERSTANDING PHYSIOLOGICAL CHANGES IN CHRONIC HEART FAILURE Haemodynamic adaptive mechanisms in the maladaptive response to heart failure}

The fall in cardiac output experienced in heart failure is detected by stretch and pressure receptors at various sites within the arteries, great veins and cardiac chambers. The underactivation of these receptors leads to a physiological response mediated through the sympathetic nervous system, the renin-angiotensin-aldosterone system and via vasopressin release from the hypothalamus. The effects of this activation lead to sodium and subsequent water retention, vasoconstriction in the renal and systemic circulation, and cardiac stimulation with increased inotropy and chronotropy. ${ }^{1}$ In healthy individuals these mechanisms are effective for restoring and maintaining cardiac output, but when ineffective they become chronically activated and maladaptive in a number of different ways.

A reduction in renal blood flow leads to increased sodium reabsorption in an attempt to increase circulating volume. This leads to fluid and electrolyte imbalance and increases total body fluid, increasing cardiac filling and aggravating systemic and pulmonary congestion. In addition, in response to a fall in renal plasma flow the efferent glomerular arteriole vasoconstricts in order to increase glomerular pressure and maintain glomerular filtration rate. This increases the potential for reabsorption of sodium and by reducing renal blood flow impairs urine output. Angiotensin II mediates this effect.

Systemic vasoconstriction is effective at maintaining cardiac output in a healthy person by decreasing the capacity of the circulation and increasing effective circulating volume. The sympathetic nervous system is mainly responsible for this effect through the stimulation of $\alpha 2$ adrenergic receptors through norepinephrine. Other vasoconstrictors include angiotensin II, vasopressin and endothelins. In chronic heart failure the increase in systemic vascular resistance reduces cardiac output further as after-load increases.

The body's response to heart failure producing sympathetic activation is the same as its response

Abbreviations: ACE inhibitor, angiotensin-convertingenzyme inhibitor; BNP, B-type natriuretic peptide 
to haemorrhagic shock or to exercise. From a cardiac perspective this leads to an increase in heart rate, inotropy and lusitropy (relaxation) produced by $\beta 1$ adrenergic receptor activation. In heart failure the subsequent response is insufficient to prevent persistent activation of the autonomic nervous system as cardiac output remains low. The chronic activation leads to down-regulation of the $\beta 1$ adrenergic receptors and reduction in cardiac responsiveness as well as having several different effects on cellular and molecular mechanisms.

\section{Cellular and molecular mechanisms of myocardial dysfunction \\ Excitation-contraction coupling and heart failure}

Excitation-contraction coupling is the chain of processes coupling the arrival of a nerve impulse at the motor end plate to contraction of the filaments of the sarcomere. Cardiac muscle contraction, like other skeletal muscles in the body, is controlled primarily by the passage of calcium into and out of myocytes and also via movement of calcium between the sarcoplasmic reticulum and the cell cytosol causing an increase in cell cytoplasmic calcium concentration and subsequent myocardial contraction. This process is a complex set of steps controlled by a number of cell messengers and receptors. The most important of these are outlined briefly below in order to illustrate the way in which heart failure affects these mechanisms.

The voltage dependent L-type calcium channel is positioned on the myocyte cell membrane (sarcolemma) and is the first point of entry for calcium into the cell. It is responsible for the slow influx of ions through the cell membrane that eventually leads to the more rapid calcium release from the sarcoplasmic reticulum, triggering myocardial contraction. Inside the myocyte calcium release from the sarcoplasmic reticulum is mediated primarily by the ryanodine receptor, triggered by the slow influx of calcium from the L-type calcium channels. It is this large increase in cytosolic calcium concentration which leads to the required conditions for calcium to interact with troponin $\mathrm{C}$ and cause contraction. The sympathetic nervous system, either by direct neuronal activation or via neurohormones, can cause an inotropic effect by increasing calcium influx through the L-type channels, producing greater release of calcium inside the cell and hence more forceful contraction. At the other end of the process cardiac muscle relaxation is mediated through either reuptake of cytosolic calcium ions via the sarcoplasmic reticulum calcium/ATPase pump or efflux mediated by sarcolemmal sodium/calcium exchangers out of the cell. The sarcoplasmic reticulum/ATPase pump is regulated by its inhibitory subunit phospholamban which when activated prevents calcium ion movement back into the sarcoplasmic reticulum.

There is broad consensus that disturbed sarcoplasmic reticulum function is the main mechanism for altered excitation-contraction coupling in heart failure. Intracellular calcium levels are generally lower in heart failure and can be explained by inadequacy of the sarcoplasmic reticulum/ATPase pump to load the sarcoplasmic reticulum with calcium, reducing the force of contraction. Reduced sarcoplasmic reticulum/ATPase pump expression has been shown in the context of decreased ventricular filling, ventricular dysfunction and heart failure. Increased phospholamban quantity or activation is also a potential target for reduced inotropy and mutations in phospholamban associated genes have been identified in subjects with cardiomyopathy. Maintenance of the complex interaction between L-type calcium channels and the ryanodine receptor is important in preventing heart failure. Increased ryanodine receptor activity in the context of heart failure is thought to be a compensatory mechanism for maintaining cardiac contractility. Calcium leak from the sarcoplasmic reticulum may be important in cardiac failure and, if so, ryanodine receptor activity could be a target for treatment. There is some evidence that $\beta$-blockers improve ryanodine receptor function. Mutations in ryanodine receptor genes are linked with arrhythmogenic right ventricular dysplasia and catecholaminergic ventricular tachycardia.

\section{Cardiovascular receptor signalling and changes with heart failure}

Adrenergic receptors are present in many different tissues throughout the body. Their importance within the cardiovascular system has been acknowledged since their early classification into $\alpha$ and $\beta$ subgroups. Both subgroups are present in the heart with the $\beta$-receptors playing the leading role. $\beta$ Receptors have since been categorised into four subgroups with $\beta 1$ and $\beta 2$ receptors being the most influential within the cardiovascular system. The $\beta 1$ subtype is expressed predominantly in cardiac tissue and activation of the receptor leads to an increase in cardiac output through inotropic and chronotropic affects as well as through greater relaxation velocity. Of the two types of $\alpha$-receptor characterised, $\alpha \mathrm{l}$ is the most significant in the heart. Activation of this receptor via the phospholipase $\mathrm{C}$ pathway can cause release of calcium directly from the sarcoplasmic reticulum, which leads to an increase in inotropy.

The change in cardiac adrenergic receptor concentrations with disease provides further insight into the molecular mechanisms underpinning cardiac failure. Stimulation of the sympathetic nervous system in response to poor cardiac output leads to an increase in plasma levels of neurohormones which stimulates $\beta$-receptors. This is advantageous as it triggers the heart to increase cardiac output in the short term. However, chronic sympathetic stimulation has a negative effect on cardiac receptor concentration. Indeed, when exogenous catecholamines are administered within an intensive care setting deactivation of receptors can occur in a only a few minutes, reducing the clinical effects observed. The overall effect of this fall in receptor quantity leads to a reduction in the sensitivity of cardiac tissue to sympathetic stimulation, implying that when cardiac output falls further the heart does not respond in an appropriate manner, potentially making the patient more symptomatic.

From a therapeutic perspective, appreciation of the effects that chronic sympathetic stimulation has on cardiac tissue enables us to understand why the use of $\beta$-blockers is beneficial. By reducing sympathetic stimulation the concentration of receptors can be preserved, enabling the heart to maintain its response to physiological changes and thus reducing symptoms.

\section{Inflammation and heart failure}

The impetus for understanding the role that the inflammatory cascade has on heart failure comes from the observation that in septicaemia cardiac function can be impaired. The reduction in function is not only related to myocardial damage by the infecting pathogen but is also potentially due to the pathophysiological response of the body's immune system. When we consider viral myocarditis, it is likely that there are different phases in which myocardial dysfunction can occur. The first phase is related to myocardial cellular damage and cell death directly related to the virus. Once the body's innate immune system becomes involved with eradicating the virus a different phase starts. It is possible that a pathological immune response could lead to autoimmunity and the development of heart failure. Subsequent to this in a third phase, once the damage has been caused, the mechanisms involved in recovery of the 
ventricle can become disordered, leading to inappropriate remodelling of cardiac tissue.

The starting point for discussing the role of inflammation in heart failure centres on the mechanism by which the inflammatory cascade can be induced. The way in which this occurs is not fully understood at present, but it is likely that neurohormones, cytokines, pathogens or free radical production through oxidative stress activates genetic transcription factors that lead to expression of pro-inflammatory mediators including TNF $\alpha$, interleukin-1 (IL-1) and interleukin-6 (IL-6) among others. One such transcription factor has been identified and is known as NF- $\kappa \beta .^{2}$

The effect of these cytokines has been investigated in animal studies and we are beginning to understand the role that this may have in causing heart failure. Overexpression of TNF $\alpha$ messenger RNA correlates with an increase in left ventricular volume and a reduction in left ventricular function. This effect may be caused by disrupting the L-type channels involved in calcium influx into the cell as discussed elsewhere in this article. TNF $\alpha$ also causes myocyte hypertrophy and apoptosis suggesting a role in pathological repair mechanisms and may be responsible for uncoupling $\beta$-adrenergic receptors and reducing the heart's sensitivity to circulating neurohormones. When IL- I is injected into mice myocarditis worsens, but conversely it improves when a receptor antagonist is administered. In addition, the IL- 1 type 1 receptor is crucial for the production of autoimmune $\mathrm{T}$ cells (CD4+) implicating a possible role in the development of an auto-reactive component to cardiac failure. IL-6 suppresses inflammation and inhibits production of TNF $\alpha$ and IL- 1 and also plays a role in apoptosis and cardiac hypertrophy. ${ }^{3}$

\section{Natriuretic peptides}

The heart itself produces a neurohormone. B-type natriuretic peptide (BNP) is a 32 amino acid polypeptide secreted by the ventricles of the heart as a response to increased wall tension from ventricular volume expansion and pressure overload. In addition to natriuresis, BNP has a vasodilating effect. Other natriuretic peptides in the group are A and C type peptides. Ventricular expression of BNP is increased with heart failure, and elevated levels of BNP are detectable in the plasma. Near patient testing kits are available for BNP and are used to diagnose heart failure. A high plasma level may indicate an adverse prognosis in children with heart failure. ${ }^{4}$ The value of $\mathrm{BNP}$ as an intravenous therapy in heart failure remains unproven.

\section{THE CLINICAL MANAGEMENT OF HEART FAILURE}

The basis for the therapeutic management of heart failure is derived from trials predominantly performed in adults. A treatment protocol has been published by the American College of Cardiologists and the American Heart Association and involves the use of diuretics, angiotensin-converting-enzyme (ACE) inhibitors, digoxin and $\beta$-blockade. ${ }^{5}$ For patients who fail to respond to these treatments the use of intravenous inotropes, mechanical support and cardiac transplantation are the subsequent forms of therapy. The strategy is similar within our paediatric practice even though the reasons for cardiac failure are not related to ischaemic heart disease as is common in adults. In the next sections of this article we will discuss the use of the key drugs and their justification as therapy for heart failure.

\section{Diuretics}

Diuretics, particularly the loop diuretics, are the first line of treatment for patients with congestive cardiac failure and have been particularly good at relieving symptoms. The use of these agents has never led to evidence of reduced mortality in adults and their use in children has never been subjected to a randomised trial other than for safety reasons. The main classes of diuretics used in children are the loop (eg, furosemide), mineralocorticoid/glucocorticoid receptor antagonists (eg, spironolactone) and thiazide diuretics (eg, metolazone). The loop diuretics are particularly good at relieving symptoms and signs of congestion but have the potential to exacerbate the maladaptive processes involved in chronic heart failure.

By blocking sodium reabsorption and decreasing the intravascular fluid volume they have the potential to induce a vicious circle of sympathetic and renin-angiotensin-aldosterone system activation which may make the scenario worse. For this reason it is imperative that they are used in combination with agents that interrupt the maladaptive responses, preferable an ACE inhibitor or a $\beta$-blocker.

In contrast to the general equivocal effect that diuretics have on prognosis, spironolactone (a mineralocorticoid/glucocorticoid receptor antagonist) has been shown to produce a $30 \%$ reduction in mortality and a $35 \%$ reduction in hospitalisations in adults with severe heart failure. ${ }^{6}$

As diuretics are the mainstay of anti-failure medication, it is important to realise that diuretic resistance is a common problem. The mechanisms for this resistance may be due to a number of different factors. Fluid overload and mesenteric congestion may prevent diuretic agents from being absorbed enterally, making an intravenous route more suitable for patients with severe oedema. Most diuretics act by blocking co-transporter ion channels within the tubules of the nephron. If the cardiac output is low, renal blood flow may be insufficient to provide an effective concentration of the diuretic in the nephron and hence a poor response to treatment results. This is particularly important when we consider that part of the maladaptive response to heart failure is vasoconstriction of the efferent arteriole and a reduction in effective renal plasma flow. Administration of low dose dopamine may help to improve renal blood flow and increase the efficacy of diuretic agents but obviously requires hospital admission and central venous access. In addition, the mechanism of action for loop diuretics involves them being bound to plasma albumin. Many children with heart failure have hypoalbuminaemia due to high metabolic demands and it is important that this is recognised when diuretic resistance is encountered. In order to overcome resistance it is important that adjunctive agents are used, particularly those that have a direct effect on reducing the maladaptive processes involved in the perpetuation of chronic heart failure. Addition of intermittent doses of metalozone can provoke effective diuresis and bumetanide is reported to have improved gastrointestinal absorption when the bowel is oedematous.

\section{ACE inhibitors}

The benefit produced by inhibiting the conversion of angiotensin I to angiotensin II is fundamental to the management of cardiac failure. The principal action through which ACE inhibitors improve survival and reduce symptoms derives from their ability to target raised filling pressures, reduced cardiac output and increased systemic vascular resistance through vasodilatation and after-load reduction. Knowledge of the mechanism of action associated with these benefits is gained through understanding the haemodynamic maladaptive strategies discussed earlier. In addition to blocking production and hence the adverse effects of angiotensin II, ACE inhibitors also potentiate the actions of bradykinin which has natriuretic properties and is responsible for the cough observed when starting captopril. This latter effect is not seen with angiotensin II receptor antagonists, such as losartan, and they are an alternative when cough is troublesome. In animal studies ACE inhibitors also prevent myocardial thickening, fibrosis and fetal 
gene modulation, all of which lead to an increase in the extracellular matrix within the left ventricle and associated adverse "remodelling". 7

Limited studies have been performed in children to investigate the benefits of ACE inhibitors; some have demonstrated a reduction in end systolic and end diastolic volumes and others have shown improved survival. ${ }^{8}$ The routine use and clear benefits extrapolated from large adult trials make a randomised control trial of the benefits of ACE inhibitors in children unnecessary and some would say unethical.

\section{Digoxin}

The use of cardiac glycosides in heart failure has become more popular over the last few years, in conjunction with data from the adult literature advocating their benefits. Digoxin affects the heart by inhibiting the energy production required by the membrane protein responsible for sodium and potassium exchange in cells. The resultant increase in intracellular sodium concentration leads to an increase in calcium ions available to the sarcoplasmic reticulum and hence increases myocardial contractility. Digoxin, as well as having inotropic properties, affects chronotropy by slowing the heart rate and reducing sympathetic activation through a reduction in plasma norepinephrine, renin and aldosterone concentrations, all of which perpetuate the exaggerated sympathetic response. ${ }^{10}{ }^{11}$

There is no evidence that digoxin administration is beneficial in children with dilated cardiomyopathy and the rationale for its use is extrapolated from the adult literature in patients with mild to moderate heart failure due to ischaemic heart disease or dilated cardiomyopathy. The largest trial showed that low dose digoxin with plasma levels of $0.5-0.9 \mathrm{ng} / \mathrm{ml}$ reduced mortality and the incidence of hospital admission, but at higher doses there is no effect on mortality. ${ }^{12}$ In our population of patients low dose digoxin is used in some children with chronic failure and can facilitate introduction of $\beta$-blockers.

\section{B-Blockers}

The increased administration and beneficial effects of $\beta$-blocker use is a prime example of how further understanding of the extended paradigms involved in cardiac failure influences clinical management. This has been discussed previously in this article, but in brief the long-term use of $\beta$-blockers prevents abnormal remodelling and potentially reverses the downregulation of adrenergic receptors seen with prolonged sympathetic activation.

A number of different classes of $\beta$-blocker have been evaluated for heart failure, all with differing effects on $\beta$ subtypes. Some newer drugs have further pleiotropic effects by reducing oxidative stress. Several important adult studies have shown the beneficial effects of $\beta$-blockers and overall results show a $30 \%$ reduction in mortality and a $40 \%$ reduction in hospitalisation. ${ }^{13}{ }^{14}$ Once again there is limited evidence for the benefits of $\beta$-blocker therapy in children, but authors have begun to focus on the potential benefits of $\beta$-blocker use. ${ }^{15}$

Introduction of $\beta$-blockers is best deferred until symptoms of congestive heart failure are controlled. From our own institutional experience we have noted that children with severe congestive heart failure who are started on $\beta$-blockers are unlikely to tolerate the reduction in sympathetic drive. However, unlike other $\beta$-blockers, carvedilol can be used in more severe myocardial failure, probably because of the vasodilating effects from its additional $\alpha$-blocking effect, although it still needs to be introduced cautiously.

\section{Oral phosphodiesterase inhibitors}

Given the evidence for $\beta$-blockers, the use of inotropic agents in the management of chronic heart failure seems counterintuitive. The use of digoxin is outlined above and is controversial, but it may have a role. Perhaps more controversial is the use of oral phosphodiesterase inhibitors such as enoximone. The greatest concern is that there is an increased risk of death, probably from arrhythmia. ${ }^{16}$ The risk appears greater than with low dose digoxin and it is likely that a large part of the improved survival with $\beta$-blockers is a result of their anti-arrhythmic effect. However, a few paediatric centres continue to use oral enoximone. While it cannot be recommended, there may be some exceptional circumstances in which it could be justified, for example when trying to wean a patient from intravenous inotropes (so far the evidence is that oral enoximone has limited success in this area) or when trying to establish $\beta$-blocker therapy (a trial is ongoing with regard to this). It would be wise to be explicit about the risks when using this medication.

\section{Anticoagulation}

Children with chronic heart failure often need anticoagulation. The risk of thrombus formation in a dilated ventricle is substantial, and the risk is increased as contraction becomes more impaired and dilatation increases. In restrictive cardiomyopathy the large atria are susceptible to thrombus formation. In older children warfarin (coumarin) is advisable, but in infants aspirin is often used as monitoring of the INR can be difficult in small children.

\section{Stem cell therapy}

The possibility of using stem cells in chronic heart failure is attractive. In dilated cardiomyopathy myocytes are replaced by fibrous tissue. Direct injection of stem cells into the myocardium or into the coronary circulation has been used in adults with heart failure. ${ }^{17}$ The success may be dependent on the type of stem cell used. However, at present stem cell usage has been confined to acute infarction. It is possible that acute inflammatory signals may be needed to allow stem cells to develop. This may not be present in the chronically failing ventricle and the role of this therapy remains unknown in chronic heart failure.

\section{The management of chronic heart failure in restrictive physiology}

This review has concentrated on the management of heart failure in children with systolic impairment. Restrictive cardiomyopathy is a rare heart muscle disease where the impaired diastolic function results in pulmonary hypertension and heart failure. The disease is often aggressive and early referral for transplantation opinion is the accepted practice. ${ }^{18}$ The management of heart failure in this setting would have some similarities to that outlined earlier. Anticoagulation is advisable and symptoms should be relieved by gentle diuresis (care should be taken not to lower the filling pressures excessively or cardiac output will fall). Paediatric hypertrophic cardiomyopathy with restrictive filling may present with symptoms of heart failure in infants, particularly in Noonan syndrome. $\beta$-Blockers such as propranolol appear useful in this setting and have been used in very high doses. In older children symptoms of heart failure in hypertrophic cardiomyopathy are rare.

\section{Transplantation and tertiary services for heart failure}

There are approximately 100 cases of new onset heart failure in children/year in the UK and Ireland (British Congenital Cardiac Association 2005, R Andrews, personal communication). Therefore, to maintain expertise it seems wise that cases are concentrated in a few tertiary clinics run by paediatric cardiologists with an interest in heart failure and paediatricians are likely to be principally involved in shared care rather than as the primary clinician. 
Cardiac transplantation is also concentrated in a few tertiary centres. It remains the ultimate treatment for chronic heart failure and approximately 20-25 heart transplants are performed at Great Ormond Street Hospital each year, which means it has of the largest paediatric heart transplant programmes in the world.

\section{CONCLUSION}

A greater understanding of the maladaptive responses to chronic heart failure has enabled targeted therapy to be introduced with consequent improvement in symptoms, reduction in hospitalisation and lower mortality.

\section{Authors' affiliations \\ Matthew Fenton, Michael Burch, Department of Cardiology, Great \\ Ormond Street Hospital, London, UK \\ Competing interests: None.}

\section{REFERENCES}

1 Katz AM. The hemodynamic defense reaction. In:Heart failure:pathophysiology, molecular biology and clinical management. Philadelphia: Lippincott Williams \& Wilkins, 2000:109-52.

2 Frantz $\mathrm{S}$, Fraccarollo D, Wagner $\mathrm{H}$, et al. Sustained activation of nuclear factor kappa Band activator protein 1 in chronic heartfailure. Cardiovasc Res 2003;57(3):749-56.

3 Hardarson HB, NE, Vallejo JG. Inflammatory mediators in heart failure. In: Chang, AC, Towbin J, eds. Heart failure in children and young adults. Philadelphia: Saunders Elsevier, 2006.

4 Price JF, Thomas AK, Grenier M, et al. B-type natriuretic peptide predicts adverse cardiovascular events in pediatric outpatients with chronic left ventricular systolic dysfunction. Circulation 2006;114(10):1063-9.

5 Hunt SA, Abraham WT, Marshall HC, et al. ACC/AHA 2005 Guideline Update for the Diagnosis and Management of Chronic Heart Failure in the Adult: a report of the American College of Cardiology/American Heart Association Task Force on Practice Guidelines (Writing Committee to Update the 2001 Guidelines for the Evaluation and Management of Heart Failure): developed in collaboration with the American College of Chest Physicians and the International Society for Heart and Lung Transplantation: endorsed by the Heart Rhythm Society. Circulation 2005; 1 12(12):e 154-235

6 Pitt B, Zannad F, Remme WJ, et al. The effect of spironolactone on morbidity and mortality in patients with severe heart failure. Randomized Aldactone Evaluation Study Investigators. N Engl J Med 1999;341(10):709-17

7 Wambolt RB, Henning SL, English DR, et al. Regression of cardiac hypertrophy normalizes glucose metabolism and left ventricular function during reperfusion. J Mol Cell Cardiol 1997;29(3):939-48.

8 Lewis $A B$, Chabot $M$. The effect of treatment with angiotensin-converting enzyme inhibitors on survival of pediatric patients with dilated cardiomyopathy. Pediatr Cardiol 1993;14(1):9-12.

9 Stern H, Weil J, Genz T, et al. Captopril in children with dilated cardiomyopathy: acute and long-term effects in a prospective study of hemodynamic and hormonal effects. Pediatr Cardiol 1990;11(1):22-8.

10 Kaye DM, Lambert GW, Lefkovits J, et al. Neurochemical evidence of cardiac sympathetic activation and increased central nervous system norepinephrine turnover in severe congestive heart failure. J Am Coll Cardiol 1994;23(3):570-8.

11 Covit AB, Schaer GL, Sealey JE, et al. Suppression of the renin-angiotensin system by intravenous digoxin in chronic congestive heart failure. Am J Med 1983;75(3):445-7.

12 Ahmed A, Rich MW, Love TE, et al. Digoxin and reduction in mortality and hospitalization in heart failure: a comprehensive post hoc analysis of the DIG trial. Eur Heart J 2006;27(2): 178-86.

13 Bristow MR, Gilbert EM, Abraham WT, et al. Carvedilol produces dose-related improvements in left ventricular function and survival in subjects with chronic heart failure. MOCHA Investigators. Circulation 1996;94(11):2807-16.

14 Packer M, Fowler MB, Roecker EB, et al. Effect of carvedilol on the morbidity of patients with severe chronic heart failure: results of the Carvedilol Prospective Randomized Cumulative Survival (COPERNICUS) study. Circulation 2002;106(17):2194-9.

15 Blume ED, Canter CE, Spicer R, et al. Prospective single-arm protocol of carvedilol in children with ventricular dysfunction. Pediatr Cardiol 2006;27(3):336-42.

16 Uretsky BF, Jessup $M$, Konstam MA, et al. Multicenter trial of oral enoximone in patients with moderate to moderately severe congestive heart failure. Lack of benefit compared with placebo. Enoximone Multicenter Trial Group. Circulation 1990;82:774-80.

17 Schachinger V, Erbs S, Elsasser A, et al. Intracoronary bone marrow-derived progenitor cells in acute myocardial infarction. N Engl J Med 2006;355(12):1210-21.

18 Fenton MJ, Chubb H, McMahon AM, et al. Heart and heart-lung transplantation for idiopathic restrictive cardiomyopathy in children. Heart 2006;92(1):85-9.

International Forum on Quality and Safety in Health Care

23-25 April 2008

Le Palais des Congrès de Paris

Call for Abstracts: Submission deadline 3 October 2007

Why submit an abstract?

- Your achievement showcased to key international opinion leaders

- Communicate your organisation's work

- Enhance your organisation's profile

- Network with others in your area/field

- Share your success, learn from your failures

For more details and to submit your abstract online visit internationalforum.bmj.com 